\title{
REVIEW OF CCD PARALLAX MEASUREMENTS
}

\author{
C.C. DAHN \\ U.S. Naval Observatory, Flagstaff, AZ USA
}

\begin{abstract}
Several groups, using 1-m to 2-m telescopes on 'visitor' arrangements, have employed CCDs to measure stellar trigonometric parallaxes with precisions in the range $\pm 2-5$ mas. More intensive observing efforts now routinely achieve sub-mas results with the best obtained to date in the $\pm 0.3-0.5$ mas range. Selective dimming of bright target stars using neutral density spots now permit CCD parallax measures of stars as bright as $\mathrm{R} \sim 4$.
\end{abstract}

\section{Introduction}

Until recently, the determination of trigonometric stellar parallaxes has remained the province of a handful of institutions with telescopes (primarily long-focal-length refractors) and plate measuring equipment essentially dedicated to this work. The demonstration that a general purpose reflecting telescope equipped with a CCD detector could produce high quality parallax determinations (Monet \& Dahn 1983), along with the widespread availability of direct imaging CCD cameras at a majority of observatories, has dramatically altered this arrangement over the past decade. Today there are at least seven groups - varying in size from a single astronomer to collaborations of 10 to 15 investigators - engaged in CCD parallax measures. The present report summarizes the current activity in CCD parallax work throughout the astronomical community.

\section{Synopses of Six Non-USNO CCD Parallax Efforts}

All groups known to be making CCD parallax measures were queried for information regarding their efforts - including the focus/scope of the program (e.g., numbers of and types of stars targeted), the telescope and instrumen-

19

T.R. Bedding et al. (eds.),

Fundamental Stellar Properties: The Interaction between Observation and Theory, 19-24.

(C) 1997 IAU. Printed in the Netherlands. 
tation (CCD camera) employed, the access to observing time, the formal internal precision of the relative parallaxes measured, and what, in their opinions, prevented them from obtaining even better results. All kindly responded and were more than generous in sharing information about their work. Very abbreviated summaries of these responses follow.

\subsection{UNIV. VIRGINIA \& SIDING SPRING OBS. CCD PARALLAX PROG.}

Collaborators P. Ianna (PI), R. Patterson, and M. Begam are employing the Siding Spring 1-m (scale: 25.8 arcsec $\mathrm{mm}^{-1}$ ) along with an EEV88530 $2 \mathrm{~K} \times 1 \mathrm{~K} \mathrm{CCD}\left(22.5 \mu\right.$ pixels, giving 0.58 arcsec pix $^{-1}$ image sampling). Normally only a $700 \times 700$ portion of the chip is employed providing a $\sim 6.8 \times 6.8$ arcmin field of view. The Cousins $\mathrm{R}$ or I bandpasses are employed. Telescope access is by quarterly application and an average of 1 week per month of gray/bright time has been received. Targets include 130 stars from the Third Catalogue of Nearby Stars (CNS3) plus 100 secondary, fainter stars from the LHS Catalogue. Based on experience from 1985 to the present, $\mathrm{Pi}(\mathrm{rel})$ precisions of $\pm 1-2$ mas are being achieved for brighter stars $(\mathrm{V} \sim 11.4-15.0 ; \mathrm{R} \sim 10.5-13)$ with $>50$ frames spanning $>1.5$ years. For fainter stars $(\mathrm{V} \sim 15-21$; I 14-17), precisions of $\pm 2-5$ mas are obtained with $>30$ frames spanning $>2.0$ years. (See also Ianna 1993.)

\subsection{UNIV. OF CHILE CCD PARALLAX PROGRAM AT CTIO}

Collaborators C. Anguita (PI), P. Loyola, and M.T. Ruiz employ the CTIO 1.5-m (scale: 10.0 arcsec $\mathrm{mm}^{-1}$ ) and CCD cameras with either Tek1K or Tek2K chips (both thinned, back-side illuminated; $24.0 \mu$ pixels, giving 0.24 arcsec $\mathrm{pix}^{-1}$ image sampling). Telescope access is by semester application and 3 or 4 nights every 3 months (generally close to New Moon) have been received. A "Broad $\mathrm{R}$ " bandpass is used and the field of view employed is $\sim 4.1 \times 4.1$ arcmin (dictated by the Tek1K field). Targets include 4060 cool WDs (from Ruiz's proper motion survey) for improving the WD LF, 3 PN central stars and 18 hot or late-type subdwarfs. All targets are faint $(15<\mathrm{R}<19)$. Based on experience from 1985 to the present, $\mathrm{Pi}(\mathrm{rel})$ precisions of \pm 1-2 mas are being achieved for well-configured, 8+ star reference star frames; otherwise, $\pm 2-3.5$ mas. A limitation mentioned is that telescope scheduling constraints necessitate their mixing data from two different CCD cameras. (See also Ruiz et al. 1990.)

\subsection{TORINO OBSERVATORY CCD PARALLAX PROGRAM}

This collaboration includes M. Lattanzi and R. Smart (Co-PIs), along with B. Bucciarelli, R. Casalegno, G. Chiumiento, R. Drimmel, H. Eichhorn, F. 
Massone, F. Morale, A. Spagna; plus Program Support from A. Ferrari, L. Lanteri, R. Morbidelli, F. Porcu, and F. Racioppi. The Torino 1.05-m (scale: $20.7 \mathrm{arcsec} \mathrm{mm}^{-1}$ ) is employed with an EEV $1246 \times 1155$ CCD (thick, frontside illuminated; $22.5 \mu$ pixels giving 0.47 arcsec $_{\text {pix }}{ }^{-1}$ image sampling). A Cousins I bandpass is used and the field of view is $10.0 \times 9.0$ arcmin. Allotment of observing time consists of all evening and morning hours of $\sim 50 \%$ of the nights - plus "some midnight hours." Of the 140 targets, 40 are bright $(\mathrm{I}<11)$. The program stars include: a $10 \%$ overlap with the USNO Parallax Program; $30 \%$ of specific interest to the Torino collaborators (Miras, PN central stars, WDs); $20 \%$ from CNS3; $25 \%$ T Tauri and/or flare stars; $15 \%$ misc. Since observations only started in 1995 , the achievable $\mathrm{Pi}$ (rel) precision is still unknown; but \pm 2 mas from $30-40$ frames is anticipated. Experiments with a neutral density spotted I band filter to provide $\sim 5$ mag of selective target star dimming look promising.

\subsection{CURRENT CCD PARALLAX EFFORTS AT LA SILLA}

Two groups are currently carrying out CCD parallax determinations using the Danish 1.54-m telescope at La Silla (scale: 18.8 arcsec $\mathrm{mm}^{-1}$ ). Access to this telescope is by semester application and both groups are employing a Gunn i bandpass.

The first collaboration - involving M. Hawkins (PI), C. Ducourant, H. Jones, and M. Rapaport - is employing a Tek1K CCD $(24.0 \mu$ pixels, giving 0.45 arcsec pix $^{-1}$ and $\mathrm{a} \sim 7.7 \times 7.7$ arcmin field of view). Targets are $\sim 30+$ very red, low-mass stars and brown dwarf candidates identified from the deep UK Schmidt plate stack in ESO/SERC field 287 at $21^{\mathrm{h}} 28^{\mathrm{m}},-45$ (1950). $\mathrm{Pi}(\mathrm{rel})$ precisions of $\sim \pm 4$ mas are anticipated but there is concern about inadequate observing time. At present the project is receiving $3 \mathrm{hrs}$ (evening or morning) on 10 nights every 6 months. Preliminary results have been submitted for publication (Hawkins et al. 1996).

The second collaboration - involving T. Forveille (PI), F. Delfosse, F. Crifo, and F. Epchtein - is employing the DFOSC focal reducing camera which provides $\sim 0.4$ arcsec pix $^{-1}$ image sampling. The targets are $\sim 20$ very red stars identified in the DENIS survey. Allotted observing time amounts to 3 runs of 2 nights each per semester near Quarter Moon. $\mathrm{Pi}(\mathrm{rel})$ precisions of $\sim 3-5$ mas are anticipated but there is concern about possible flexures in the reimaging system.

\subsection{TINNEY CCD PARALLAX MEASURES AT PALOMAR \& LA SILLA}

An important example of a single investigator carrying out CCD parallax determinations targeted at a very specific scientific problem - that of elucidating the nature of very-low-mass star and brown dwarf candidates - is 
the work of C. Tinney. Although no longer active, these efforts illustrate the important contributions that "limited" projects carried out at 'visitor' facilities can accomplish. In both instances, telescope access was by semester applications and the Gunn i bandpass was employed.

The Palomar 1.5-m (scale: 15.5 arcsec $\mathrm{mm}-1$ ) was used with two different Tek1K CCDs (the first thick, front-side illuminated; the second thinned, back-side; both $24 \mu$ pixels, giving 0.37 arcsec pix $^{-1}$ image sampling and a $\sim 6.3 \times 6.3$ arcmin field of view - the latter noted as only "sufficient"). The project received 5 runs of gray-dark observing time a year between September 1990 and December 1993. Parallax precisions in the range $\pm 4-5$ mas were achieved on targets typically I $\sim 14.5-17.5$ from $3-4$ years of data. (See Tinney et al. 1995.)

The La Silla work employed the ESO 2.2-m (scale: 17.5 arcsec $\mathrm{mm}^{-1}$ ) and the EFOSC2 reimaging system which uses a Thomson $1 \mathrm{~K} \times 1 \mathrm{~K} \mathrm{CCD}$ $\left(19.0 \mu\right.$ pixels, giving 0.33 arcsec pix $^{-1}$ image sampling and a $5.7 \times 5.7$ arcmin field of view - again, only "sufficient"). This project received 4 observing runs a year, typically at gray-dark lunar phases between May 1993 and February 1995. Typical $\mathrm{Pi}(\mathrm{rel})$ precisions were in the range $\pm 3-7+$ mas (median: \pm 4.5 mas) from 2 years of data. Concerns were expressed about flexures in the reimaging system. (See Tinney 1996.)

\section{Status of the USNO, Flagstaff CCD Parallax Program}

The USNO, Flagstaff efforts are an outgrowth of work carried out with the KPNO 4-m in 1980-1983 (Monet \& Dahn 1983). This program has evolved considerably over the ensuing years and presently can be summarized best as a set of sub-programs, each defined by a specific CCD camera employed for the observations. Current in-house collaborators include: C. Dahn (PI), H. Guetter, F. Harris, H. Harris, A. Henden, C. Luginbuhl, A. Monet, D. Monet, J. Pier, R. Stone, F. Vrba, and R. Walker. The telescope used is the USNO 1.55-m Astrometric Reflector (scale: $13.55 \mathrm{arcsec}^{-1}$ ) and access for CCD astrometry (primarily parallax work) has remained at $\sim 20$ nights per lunation, centered around New Moon.

\subsection{THE USNO TI800 PARALLAX PROGRAM}

The TI800 program, which operated from March 1983 through October 1995, has been described by Monet et al. (1992) where the results for 72 stars were presented. A total of $\sim 175$ parallax determinations have been completed with this camera and publication of the remaining results for the additional $100+$ stars is in preparation. Due to the small field of view of the TI $800 \times 800 \mathrm{CCD}(\sim 2.7 \times 2.7$ arcmin at the USNO $1.55-\mathrm{m})$ many marginal quality reference star frames were used. Nevertheless, the formal precisions 
of the $\mathrm{Pi}(\mathrm{rel})$ determinations ranged from $\pm 1.0-2+$ mas for average-topoorer reference frames, to \pm 0.7-1.0 mas for better reference frames, to $\pm 0.5-0.7$ mas for the best cases.

\subsection{THE USNO TEK2K PARALLAX PROGRAM}

Initiated in February 1992, a program employing a Tek2K CCD (thinned, back-side illuminated; $24.0 \mu$ pixels giving $0.325 \operatorname{arcsec}$ pix $^{-1}$ and an $11 \times 11$ arcmin field of view) has now become the mainstay of USNO parallax efforts. The large full-well capacity of this chip $\left(>300 \mathrm{k} \mathrm{e}^{-}\right)$provides a working astrometric dynamic range of 4.5-5 magnitudes and assures good-toexcellent reference star frames for $>95 \%$ of the targets attempted to date. Note, however, that without selective magnitude compensation - that is, the ability to selectively dim a target star while simultaneously exposing fainter reference stars - the Tek2k program, like all other CCD parallax efforts discussed thus far, is restricted to relatively faint stars; in this case to those primarily with $\mathrm{R}>12$. The $\sim 200$ stars under observation (or already completed) have been selected primarily from Luyten's Palomar Proper Motion Survey (a mixture of degenerates, late-type dwarfs and subdwarfs) but includes special interest targets such as PN central stars and very low mass stars from the work of Tinney and others. Based on solutions for fields with epoch ranges spanning $2-4+$ years, $\mathrm{Pi}(\mathrm{rel})$ precisions of $< \pm 1.0$ mas are routinely being obtained. The median $\mathrm{Pi}(\mathrm{rel})$ precision achieved for 67 completed solutions derived from 3.1-4.4 years of data (median: 4.2 years) is \pm 0.55 mas. For the best cases ( 30 completed solutions), the values range between \pm 0.28 mas and \pm 0.54 mas.

\subsection{THE USNO ND9 PARALLAX PROGRAM}

In an attempt to address the inability to target stars brighter than $\mathrm{R} \approx 12$, a second Tek2K CCD camera has been outfitted with a neutral density attenuation spot to selectively dim a target star near the center of the field. The spot is a highly uniform deposit of Inconel on an optically flat quartz substrate and is mounted $\sim 1 \mathrm{~mm}$ in front of the CCD. This spot is circular, roughly $3 \mathrm{~mm}$ in diameter $(\approx 40 \mathrm{arcsec})$ and provides about 9.0 magnitudes of attenuation. (Hence, the name "ND9.") The particular Tek2K CCD employed in this camera is only an 'Engineering Grade' device and, consequently, has several blocked columns, a number of 'charge traps' and a higher read-noise than the chip employed in the previously described system. The substrate mounting arrangement also diminishes the available field of view to approximately $9.0 \times 9.0 \mathrm{arcmin}$. Placed in operation in December 1995, this camera is being used on 68 targets, mostly in the $5.2<\mathrm{V}<9.5$ brightness range. Included are: $27 \mathrm{sdF}-\mathrm{G}$ stars, 9 Solar 
Analogs, 5 Periodic Variables (SU Cas, VZ Cnc, RR Lyr, $\eta$ Aql, $\delta$ Cep), 4 Field Horizontal Branch stars, 4 Hyades Cluster members, and 2 Mira Variables ( $o$ Cet, R Cas). Sixty-five of the 68 present targets are also in Hipparcos Input Catalogue. Several potential concerns - such as excessive scattered light and flattening of the chip response in the area behind the spot - have proved not to be serious. Preliminary reductions have been carried out on the 40 fields which have sufficient epoch coverage (354-360 days) and enough variation in parallax factor to support separation of proper motion from parallax. The results are very encouraging in that: (1) the internal $\mathrm{Pi}(\mathrm{rel})$ precisions are already down to $\pm 0.9-2.8$ mas (median: \pm 1.7 mas); and (2) where good quality independent parallax determinations are available from the Yale Parallax Catalogue, the agreement is excellent.

\section{Summary and Conclusions}

Relative trigonometric parallaxes with formal internal errors in the range $\pm 2-5$ mas are routinely being measured under 'visitor' arrangements by investigators employing 1-2-m aperture telescopes and a variety of CCD cameras. With more intensive observing programs, sub-mas results are routinely acheived and the best results to date are in the $\pm 0.3-0.5$ mas range. The absence of a facility equipped (and willing) to provide such intensive observations for Southern Hemisphere targets is still a significant deficiency for the astronomical community. On the positive side, successful magnitude compensation has now been demonstrated, permitting CCD parallax measures for stars as bright as fifth magnitude.

\section{References}

Hawkins, M.R.S., Ducourant, C., Jones, H.R.A., and Rapaport, M. 1996, MNRAS, submitted.

Ianna, P.A. 1993, in Developments in Astrometry and Their Impact on Astrophysics and Geodynamics, IAU Symp. No. 156, ed. I.I. Mueller and B. Kolaczek, 75.

Monet, D.G. and Dahn, C.C. 1983, AJ, 88, 1489.

Monet, D.G, Dahn, C.C., Vrba, F.J., Harris, H.C., Pier, J.R., Luginbuhl, C.B., and Ables, H.D. 1992, AJ, 103, 638.

Ruiz, M.T., Anguita, C., and Maza, J. 1990, AJ, 100, 1270.

Tinney, C.G. 1996, MNRAS, 281, 644.

Tinney, C.G., Reid, I.N., Gizis, J., and Mould, J.R. 1995, AJ, 110, 3014. 